\title{
Numerus surdus y armonía musical. Sobre el temperamento igual y el fin del reinado pitagórico de los números
}

\author{
Numerus surdus and musical harmony. On the equal temperament \\ and the end of the Pythagorean reign of numbers \\ Lianggi Espinoza*; Juan Redmond**; \\ Pablo César Palacios Torres ${ }^{* * *}$; Ismael Cortez Aguilera ${ }^{* * * *}$ \\ *Universidad de Valparaíso, Chile \\ lianggi.espinoza@uv.cl \\ ${ }^{* *}$ Universidad de Valparaíso, Chile \\ juan.redmond@uv.cl \\ ${ }^{* * *}$ Universidad de Valparaíso, Chile \\ pablo.palacios@uv.cl \\ ${ }^{* * * *}$ Universidad de Valparaíso, Chile \\ ismael.cortez@uv.cl
}

\section{Resumen}

El desarrollo de ideas filosóficas a lo largo de la historia ha estado acompañado en ocasiones determinantes por la intervención de instrumentos artesanales. Algunos casos paradigmáticos, como la invención del telescopio o del microscopio, evidencian que muchos enfoques filosóficos han sido el fruto de la intervención de este tipo de instrumentos. El objetivo del presente artículo es mostrar el rol determinante que tuvieron los instrumentos musicales de cuerdas con trastes en la crisis y generación de paradigmas filosóficos. En efecto, así como las observaciones de la luna con el telescopio resquebrajó más de un milenio de hegemonía aristotélica, los instrumentos de cuerda con trastes, antecesores de la guitarra, jugaron un rol central en el desmoronamiento de uno de los enfoques más influyentes de la historia de la Filosofía: el pitagorismo. Nos focalizamos en los hitos fundamentales del pitagorismo y en cómo surge, durante el siglo XVI y desde los instrumentos de cuerda con

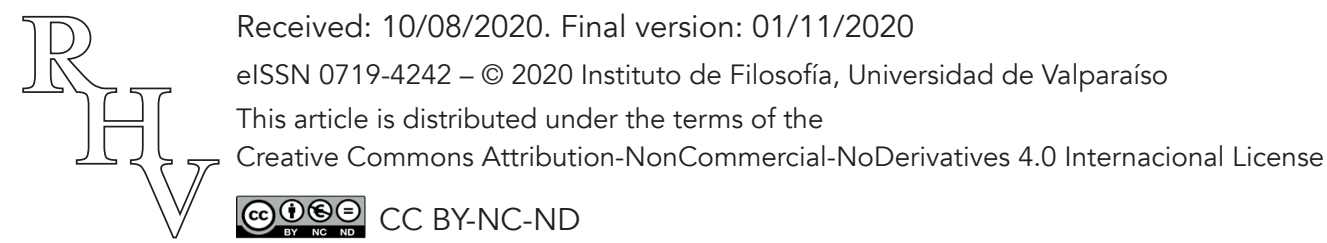


Numerus surdus y armonía musical. Sobre el temperamento igual y el fin del reinado pitagórico de los números

Lianggi Espinoza; Juan Redmond; Pablo César Palacios Torres; Ismael Cortez Aguilera

trastes, la noción matemático-musical de temperamento igual, que desde mediados del siglo XIX se establecerá como el paradigma filosófico-musical predominante de Occidente.

Palabras clave: temperamento igual, filosofía, pitagorismo, música matemática.

\begin{abstract}
The development of philosophical ideas throughout history has sometimes been assisted by the use of handcrafted instruments. Some paradigmatic cases, such as the invention of the telescope or the microscope, show that many philosophical approaches have been the result of the intervention of such instruments. The aim of this article is to show the determining role that stringed musical instruments with frets had in the crisis and generation of philosophical paradigms. In fact, just as the observations of the moon with the telescope broke more than a thousand years of Aristotelian hegemony, the fretted string instruments, predecessors of the guitar, played a central role in the collapse of one of the most influential approaches in the history of Philosophy: Pythagorism. We focus on the fundamental hallmarks of Pythagorism and on how, during the 16th century and from the fretted string instruments, the mathematical-musical notion of equal temperament emerged, which from the middle of the 19th century will be established as the prevailing philosophical-musical paradigm of the West.
\end{abstract}

Keywords: equal temperament, philosophy, pythagorism, mathematical music.

\title{
1. Introducción
}

Herencia de los pitagóricos es la idea de que la música es de naturaleza numérica. Entre los pitagóricos, además del propio Pitágoras, desde luego, cuentan como los más conocidos, de acuerdo con el catálogo de Iamblichus (1991), Epicarmo de Megara, Alcmeón de Crotona, Hipaso de Metaponto, Filolao de Crotona y Arquitas de Tarento. Para estos los sonidos musicales (en términos actuales, las notas) son números, es decir, números que suenan. Cada sonido musical es concebido como una distancia en una cuerda. Cada una de estas distancias determinan un intervalo musical al que le corresponde una razón. Esta es la idea que está por detrás de la afirmación de que la música es "números sonando". Estos números, de un modo más específico, corresponden a lo que se llama razones o ratios. Es decir, según los pitagóricos, donde hay un sonido, debe haber una proporción exacta y calculable que corresponda a tal intervalo musical ${ }^{1}$.

\footnotetext{
${ }^{1}$ En la actualidad, en matemáticas se enfatiza la diferencia existente entre una razón $(\mathrm{a} / \mathrm{b})$ y una proporción $(\mathrm{a} / \mathrm{b}=\mathrm{c} / \mathrm{d})$. En el contexto de la matemática griega no se concibe de esta manera, pues se entiende que una ratio expresa en sí misma una proporción.
}

Revista de Humanidades de Valparaíso, 2020, No 16, 137-167

(c) (1) @९ $\mathrm{O}$ CC BY-NC-ND 
Numerus surdus y armonía musical. Sobre el temperamento igual y el fin del reinado pitagórico de los números Lianggi Espinoza; Juan Redmond; Pablo César Palacios Torres; Ismael Cortez Aguilera

Uno de los intervalos musicales más importantes es la octava, a la cual le corresponde la razón o ratio de la forma 2:1. La octava es el mismo sonido, la misma nota en nuestros términos, pero en una altura mayor. De esta manera, dada una cuerda de largo y tensión invariables, la octava 'suena' en el intervalo que se determina con el punto medio de la cuerda. Otros dos intervalos musicales de gran importancia son la quinta, de ratio 3:2, y la cuarta, de ratio 4:3. Estos intervalos son los denominados sonidos puros, esto es, sonidos llanos que son agradables al oído, que suenan sin batimentos (oscilaciones). Ahora bien, debió ser un descubrimiento formidable que tales sonidos puros correspondieran a ratios tan precisas. Este hecho llevó a los pitagóricos (de acuerdo con la leyenda) a generalizar la idea de que allí donde pisamos una cuerda con el dedo y hacemos sonar cierto intervalo, solo aparece un sonido adecuado si tal intervalo corresponde a una proporción matemática. Es decir, no debería haber sonido sin ratios.

Si bien, esta correspondencia entre sonidos puros y ratios fue un gran hallazgo, se volvió infructuosa al determinar pequeños intervalos musicales como por ejemplo la división del tono. Los antiguos definieron el tono (9:8) como la diferencia entre el intervalo de quinta y el de cuarta ${ }^{2}$. La división del tono se volvió un tema significativo en la teoría musical de la antigüedad, ya que al dividirlo se puede construir todo el espectro de posibilidades sonoras para la creación de melodías. Ahora bien, al dividir el tono en dos partes iguales ${ }^{3}$, se obtenía un intervalo musical al que no le correspondía una proporción exacta, es decir, la división igual del tono no es reducible a una razón del tipo a/b, dado que es una magnitud inconmensurable. Aquí surge la interrogante ¿cómo podía haber sonidos que no tuvieran su correspondiente ratio? Este asunto puso en crisis el enfoque cuantitativo de la música de los pitagóricos y, al mismo tiempo, su metafísica numérica del mundo. Para desarrollar este asunto, comenzaremos refiriéndonos al enfoque musical de los pitagóricos.

\section{Pitagorismo en perspectiva}

Pitágoras nació en las primeras décadas del siglo VI a. C. (aproximadamente entre 570 y 490 a. C.) y vivió lo suficiente como para abarcar, según Empédocles, "todas las cosas contenidas en diez, y hasta en veinte generaciones de hombres" (Burnet 1928, 43). De acuerdo con Huffman (2018), es imposible establecer en nuestros días si cada detalle particular del universo de creaciones que se le atribuyen son realmente de él, de sus discípulos o simplemente leyendas elaboradas por sus seguidores. Sin duda se trató de un

\footnotetext{
${ }^{2}$ Sumar intervalos musicales implica, en términos numéricos, multiplicar sus ratios. A su vez, se restan intervalos dividiendo sus ratios. De esta manera, la diferencia entre el intervalo de quinta y el de cuarta es la división entre $3 / 2$ y $4 / 3$, es decir, $9 / 8$.

${ }^{3}$ Dividir en partes iguales significa dividir un intervalo musical por la mitad en términos de su sonido. Matemáticamente, esto se encuentra mediante una proporción geométrica (aritméticamente mediante el cálculo de una raíz y geométricamente con una media proporcional).
}

Revista de Humanidades de Valparaíso, 2020, No 16, 137-167

(c) $(1)(9)$ CC BY-NC-ND 
Numerus surdus y armonía musical. Sobre el temperamento igual y el fin del reinado pitagórico de los números Lianggi Espinoza; Juan Redmond; Pablo César Palacios Torres; Ismael Cortez Aguilera

personaje que despertó pasiones y por ello se le debió conceder más cosas que las que desarrolló. Las evidencias conocidas (cf. Burnet 1928) sugieren que no escribió ningún libro:

Pythagoras must have been one of the world's greatest men, but he wrote nothing, and it is hard to say how much of the doctrine we know as Pythagorean is due to the founder of the society and how much is later development. (Burnet 1928, 37)

En efecto, ninguna fuente contemporánea de Pitágoras o de los primeros doscientos años después de su muerte -incluidos Platón, Aristóteles y sus sucesores inmediatos en la Academia y el Liceo- , cita una obra de Pitágoras o da alguna indicación de que existiera alguna obra escrita por él. En general, no es fácil separar su obra del mito en torno a su figura como gran maestro. Sin embargo, parece difícil dudar que fuera el fundador de una nueva filosofía religiosa y quizás por ello se convirtió en un personaje de gran admiración. Desde luego esto debió generar muchas enemistades. En efecto, consta que luego de establecerse en Crotona alrededor del 530 a. C., fundó la Fraternidad Pitagórica que ejerció una supremacía considerable en la Magna Grecia pero que no duró mucho: Pitágoras fue desterrado a Metaponto y sus discípulos corrieron igual o peor suerte.

\subsection{La música y el saber unificador de los pitagóricos}

Si pensáramos en las razones principales del poder del mensaje de Pitágoras y el de sus discípulos, estas serían su poder integrador y su sentido unificador. Es decir, se trata de una perspectiva que lo abarca todo: la religión y la ciencia, la matemática y la música, la medicina y la cosmología, el cuerpo, la mente, el espíritu, todo capturado en una síntesis que sin duda cautivó e influyó de manera contundente a generaciones. En el pitagorismo, todos estos campos constituyen una unidad y un modo de comprenderlo es ingresando por el campo de la música. En efecto, de acuerdo con Huffman (2018), aunque muchos autores basados en ciertas fuentes ponen en duda si el origen de estas ideas es realmente de Pitágoras o de alguno de sus discípulos, la relación entre matemática y música es considerada la clave de la unidad en su perspectiva (cf. Burnet 1928). Se trata de la consideración de la relación entre los sonidos musicales y las longitudes de las cuerdas que los producen. Es decir, los intervalos musicales se deben a proporciones numéricas. Esto último es considerado por muchos como la primera reducción de la calidad a la cantidad e inicio de la ciencia tal como la conocemos en nuestros días. Este enriquecimiento de la experiencia humana, tal como lo concebían los pitagóricos, daba cuenta de una de las creencias más significativa de estos filósofos: los números eran sagrados, de naturaleza ideal y pura (espiritual diríamos en nuestros días), y por ello imperecederos. Al respecto, dice Aristóteles: 
Numerus surdus y armonía musical. Sobre el temperamento igual y el fin del reinado pitagórico de los números Lianggi Espinoza; Juan Redmond; Pablo César Palacios Torres; Ismael Cortez Aguilera

En la misma época que éstos, y aun antes que ellos, los denominados Pitagóricos, dedicándose los primeros a las matemáticas, las hicieron avanzar, y nutriéndose de ellas, dieron en considerar que sus principios son principios de todas las cosas que son. Y puesto que en ellas lo primero son los números, y creían ver en éstos -más, desde luego, que en el fuego, la tierra y el agua- múltiples semejanzas con las cosas que son y las que se generan. $(1994,89)$

De esta manera, el objetivo de la ciencia para Pitágoras y los pitagóricos, no es la indagación de lo material sino de la proporción, de la forma y de los esquemas que entretejen lo material. Esta relación fundamental fue extendida por ellos a las otras esferas de la realidad: hacia los astros, por una parte, y hacia el cuerpo y el alma del ser humano, por otra (cf. Huffman 2018). Y los puntos de apoyo para estas proyecciones eran los conceptos de armonía y catarsis. La escuela pitagórica reunía estas enseñanzas éticas en una colección de sentencias llamadas Acusmata. Las ideas pitagóricas en torno a la relación entre proporciones matemáticas, música y cuerpo humano, siguen teniendo significativa influencia en la actualidad (cf. Lindley \& Turner-Smith 1993, 11). Un testimonio de tal influencia es la persistencia en el lenguaje de términos como tónico (tono), temperamento y armonía para hacer referencia a nociones relativas a la salud física y mental de los seres humanos. Para los pitagóricos, el conocimiento musical no era solo el conocimiento de un arte sonoro, sino que, de acuerdo con su filosofía, era el conocimiento de la naturaleza última del mundo. Es decir, la música es un vehículo para la contemplación intelectual a través de los sentidos.

\subsection{Pitágoras, las matemáticas y la música}

La relación existente entre música y números es un principio fundamental dentro de la teoría musical pitagórica. Esta relación se hace evidente en la tetraktys. Literalmente "las cuatro", la tetraktys se refiere a la figura geométrica (un triángulo equilátero) formada con 10 puntos (número perfecto en el pitagorismo en el siglo quinto a. C.) y que se construye con filas de uno, dos, tres y cuatro puntos. Iamblichus informa $(1989,36)$ del siguiente acusmata: “QQué es el oráculo de Delfos? La tetraktys, que es la armonía en la que cantan las sirenas". Es la música que cantan las sirenas en el sentido de que todos los intervalos que producen sonidos puros (octava 2:1, quinta $3: 2$ y cuarta $4: 3$ ) pueden expresarse como proporciones de números $1,2,3$ y 4 .

La tradición posterior a Pitágoras le atribuye el famoso descubrimiento de que las concordancias o intervalos musicales principales (la octava, la quinta y la cuarta) corresponden las proporciones 2:1, 3:2 y 4:3, respectivamente (cf. Iamblichus 1989, 50). La única fuente temprana que asocia a Pitágoras con tales proporciones, es decir, con los números 1, 2, 3 y 4 gobernando las consonancias, es Jenócrates (Fr.9) en la Academia temprana. Pero, como señala Huffman (2018), la Academia temprana es una fuente posterior de la 
Numerus surdus y armonía musical. Sobre el temperamento igual y el fin del reinado pitagórico de los números

Lianggi Espinoza; Juan Redmond; Pablo César Palacios Torres; Ismael Cortez Aguilera

tradición, seguramente exagerada y poco fiable sobre Pitágoras. En efecto, la leyenda cuenta que Pitágoras pasó por una herrería y escuchó las concordancias en los sonidos de los martillos golpeando el yunque y descubrió que los sonidos de los martillos cuyos pesos están en la proporción 2:1, 3:2 y 4:3 estarían separados por una octava, una quinta y una cuarta, respectivamente. Desafortunadamente, esta historia es probablemente falsa dado que ninguna de las técnicas para el descubrimiento que se le atribuyen funcionaría (cf. Burkert 1972, 375). De hecho, el propio Vincezo Galilei (padre del conocido Galileo Galilei) presenta una prueba de la no correspondencia entre los pesos y los tonos musicales (Galilei 1589).

De acuerdo con la tradición pitagórica, si las ratios son una propiedad de los intervalos, entonces las mismas ratios de peso gobernando la tensión de una cuerda fija de largo inalterado deberían producir los mismos intervalos musicales. Sin embargo, Vincezo Galilei desarrolla un experimento que muestra que la proporción de pesos gobernando la tensión de una cuerda de largo fijo es diferente de la proporción de distancias en la misma cuerda de tensión fija. Al concluir esto, Galilei se pregunta lo siguiente: si la relación no se cumple para los pesos, ¿por qué debe suponerse que los intervalos musicales corresponden a ratios proporcionales? Existe alguna evidencia, según Huffman (2018), de que esta relación entre pesos y ratios ya la conocía Lasus, contemporáneo de Pitágoras, y que no era pitagórico (Burkert 1972, 377). A su vez, Garrido $(2012,130)$ plantea que Iamblichus sostuvo que el descubrimiento de las proporciones armónicas es propio de los babilónicos, de quién Pitágoras las aprendió y las llevó a Grecia.

Por tanto, puede ser que Pitágoras supiera de la relación sin haberla descubierto ni demostrado científicamente. La relación probablemente fue descubierta por primera vez por los fabricantes de instrumentos de viento (Barker 2014, 202). Así, la imagen de Pitágoras que surge de la evidencia parece sugerir que no es la de un matemático como Euclides, por ejemplo, que ofreció pruebas matemáticas rigurosas, o la de un científico, que llevó a cabo experimentos para conocer la matemática en el mundo natural. Más bien, esta imagen es la de alguien que ve un significado especial en las relaciones matemáticas que estaban en circulación en su época (cf. Huffman 2018).

\subsection{Música e inconmensurabilidad}

En el contexto de la matemática griega, se consideran como números a los números naturales, los cuales pueden ponerse en relación unos con otros formando razones ${ }^{4}$. También, los griegos estudiaron sucesiones de tres o más números formando progresiones (González 2014, 744). Ahora bien, que una razón sea conmensurable significa que existe una magnitud que es una medida común de los dos números naturales que la conforman.

\footnotetext{
${ }^{4} \mathrm{Si}$ bien, la idea de razones equivale a lo que actualmente denominamos fracciones o números racionales, los griegos no las consideraron de esta manera (González 2014, 744).
}

Revista de Humanidades de Valparaíso, 2020, No 16, 137-167

(c) $(1)(9)$ CC BY-NC-ND 
Numerus surdus y armonía musical. Sobre el temperamento igual y el fin del reinado pitagórico de los números Lianggi Espinoza; Juan Redmond; Pablo César Palacios Torres; Ismael Cortez Aguilera

Esta medida común entre dos segmentos (el máximo común divisor en términos actuales) tiene un rol protagónico en la matemática griega. En efecto, dos magnitudes inconmensurables eran aquellas para las cuales no se podía encontrar una medida común, o bien, que no se puede expresar la cantidad de veces que una está contenida en otra. Es lo que ocurre, por ejemplo, para el caso del lado y la diagonal de un cuadrado, las cuales son magnitudes inconmensurables (asimmetros, sin medida común) (González 2014, 744).

¿Cómo descubren Pitágoras o los pitagóricos que hay intervalos musicales que son inconmensurables? Esta pregunta no tiene una respuesta clara. Es decir, no consta en ninguna fuente que el propio Pitágoras hablara de este tema. Así lo sugiere, entre otros, Tannery, uno de los comentadores más importantes sobre este tema:

Le problème de la commensurabilité ou non de la diagonale du carré et de son côté n'avait, en somme, en géométrie qu'un intérêt purement théorique. Mais à la même époque [celle des premiers pythagoriciens] se posait un problème dont l'importance philosophique était au moins aussi grave (...) Entre les deux motifs, l'un géométrique, l'autre musical, qui pouvaient, dans une première moitié du cinquième siècle, sinon dès le temps de Pythagore, provoquer l'étude de la question, on ne peut prétendre à déterminer lequel fut en réalité le plus actif. Mais on ne saurait nier le rôle joué dès lors par le problème musical, et il dut au moins amener de nouvelles recherches pour une approximation aussi exacte que possible de $\sqrt{ } 2$. $(1915,85-86)$

El descubrimiento de distancias no cuantificables de acuerdo con ratios significó, en palabras de Brunschvicg $(1947,46)$ : “el fin del reinado de los números", es decir, el desmoronamiento de la naturaleza numérica de la realidad, una de las tesis fundamentales del pitagorismo. Iamblichus nos dice que los pitagóricos intentaron mantener en secreto este descubrimiento: "celui qui avait révélé ce qui concerne l'irrationalité et l'incommensurabilité, a subi le même sort [qu'Hippasse]". Esto último, luego de haber divulgado algunos secretos fue acusado de impiedad, excluido de la secta pitagórica y "périt dans les flots suite au châtiment divin" (cf. Iamblichus 1989, 39).

Los intervalos de octava, de quinta, de cuarta, y otros intervalos pitagóricos son conmensurables. Sin embargo, la imposibilidad de dividir el tono (9/8) en dos partes iguales generó una gran controversia por su inconmensurabilidad. De esta manera, surge el problema de saber si estos tipos de intervalos suenan o no. Al respecto, en el paradigma pitagórico, si la música es 'números sonando', estas distancias inconmensurables serían números sordos. Podemos interpretar sordo en el sentido de que los humanos no deberíamos escuchar sonidos provenientes de dichos intervalos de cuerda. También podríamos pensarlo como números mudos, en el sentido de que esa distancia no debería ser capaz de emitir sonido alguno. Este punto es central, pues en el sistema de división de la cuerda que usamos actualmente cada división está definida por magnitudes inconmensurables y 
Numerus surdus y armonía musical. Sobre el temperamento igual y el fin del reinado pitagórico de los números Lianggi Espinoza; Juan Redmond; Pablo César Palacios Torres; Ismael Cortez Aguilera

la razón que constituye esta división también es inconmensurable. ¿Cómo llegamos de un paradigma que niega los inconmensurables en la música al sistema actual basado en magnitudes inconmensurables? Esto lo desarrollaremos a continuación.

\section{Euclides y los inconmensurables}

La obra de Euclides de Alejandría (aproximadamente 325-265 a. C.) ocupa un lugar central en el desarrollo histórico del temperamento igual, el cual es el paradigma musical predominante en la actualidad. A continuación, explicaremos el problema de la teoría musical antigua respecto a la posibilidad de dividir el tono en partes iguales y la relación de este problema con la proposición 13 del libro VI de los Elementos de Euclides (1774). A su vez, abordaremos el extravío de los Elementos en Occidente en el siglo VI d. C. (Strunk 1950) y las repercusiones de su retorno desde el mundo árabe durante el siglo XII.

\subsection{Respecto a la división del tono en partes iguales}

El problema de la imposibilidad de dividir el tono en dos partes iguales, por su inconmensurabilidad, fue uno de los problemas centrales de los teóricos musicales griegos de la antigüedad (González 2014). Esto se expresa particularmente la Sectio Canonis, un tratado pitagórico de música del siglo III a. C. que se ha atribuido a Euclides. Según González, "este es el tratado conservado más antiguo en el que aparece explicada la teoría pitagórica de la música y es aproximadamente contemporáneo de los Elementos" (2014, $746)^{5}$. En el teorema XVI de la Sectio Canonis se afirma que "el tono no será dividido ni en dos ni en más intervalos iguales" (Garrido 2016, 50). Este principio pitagórico de la música fue sostenido por diversos teóricos musicales que suscribieron al pitagorismo, como Ptolomeo, Quintiliano y Boecio (González 2014).

La imposibilidad de dividir el tono en dos partes iguales deviene de que para los griegos la música fue una disciplina numérica. Esta imposibilidad se daba para varios intervalos musicales, no solo para el semitono. Dado que en la aritmética griega solo se consideraban los números naturales y las razones, tales magnitudes no eran consideradas números ni se desarrollaron en su aritmética. Así, la imposibilidad de concebir una magnitud inconmensurable era numérica, no geométrica. En efecto, tales magnitudes se estudiaron en la geometría griega. Un ejemplo de esto es la proposición 13 del Libro VI de los Elementos, donde Euclides (1774) brinda el método geométrico para encontrar una

\footnotetext{
${ }^{5}$ La primera parte expone unos teoremas con contenido puramente matemático, y la segunda aplica lo señalado al campo musical. Según Gonzalez $(2014,746)$, esto recuerda al mundo de los Elementos, pero con un nivel matemático más elemental y menos general”.
}

Revista de Humanidades de Valparaíso, 2020, No 16, 137-167

(c) (1) @९ CC BY-NC-ND 
media proporcional entre dos magnitudes dadas. Esta es, dado los segmentos $\mathrm{AB}$ y $\mathrm{BC}$, una magnitud $\mathrm{BD}$ que esté en proporción media entre los dos segmentos dados, es decir, que se tenga que $\mathrm{AB} / \mathrm{BD}=\mathrm{BD} / \mathrm{BC}$ (Figura 1 ).

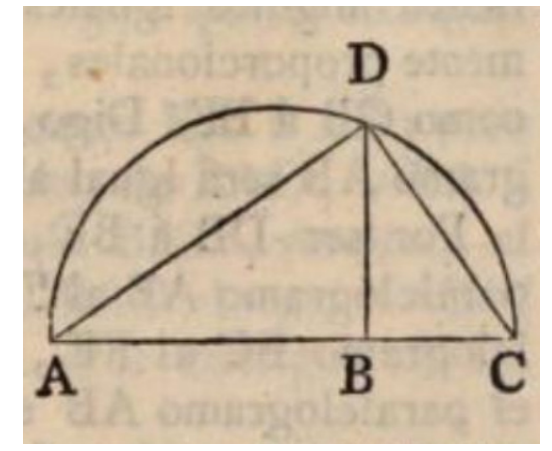

Figura 1. Construcción geométrica de la proposición 13 del Libro VI de los Elementos (Euclides 1774, 163).

La técnica de Euclides para encontrar la media proporcional BD consiste en considerar las rectas $\mathrm{AB}$ y $\mathrm{BC}$ de manera contigua, trazar la semi circunferencia inscrita en el segmento $\mathrm{AC}$ y trazar una recta perpendicular a $\mathrm{AC}$ en el punto $\mathrm{B}$, obteniendo el punto $\mathrm{D}$ como intersección entre la semicircunferencia y la recta perpendicular. El segmento resultante $\mathrm{BD}$ es la media proporcional entre $\mathrm{AB}$ y $\mathrm{BC}$. Cabe señalar que la idea subyacente en este teorema es intrínsecamente geométrica y proporcional. Es decir, se tiene que $\mathrm{BC}$ está en proporción a BD como BD lo está a BA. Esta idea puede verse en la proposición 8 del Libro VI, en donde tal proporcionalidad es evocada a través de concebir a los triángulos CBD, DBA y ACD como triángulos semejantes.

Este teorema tiene una importancia tal en la geometría euclidiana que hoy lo conocemos como "teorema de Euclides". Sin embargo, al considerar a la música como una disciplina numérica, los griegos antiguos no utilizaron el teorema de Euclides para dividir el tono u otros intervalos musicales en partes iguales (González 2014). Ahora bien, dado las dificultades prácticas que derivó el planteamiento de la imposibilidad de dividir ciertos intervalos musicales en partes iguales, en la antigüedad surgió una corriente teórica musical que postuló la posibilidad de realizar dichas divisiones. Nos referimos a Aristoxeno y sus seguidores, quienes sostuvieron que el todo podía ser dividido en medias, terceras o cuartas partes iguales (Garrido 2016) ${ }^{6}$.

No obstante, el pitagorismo fue una de las perspectivas más influyentes de la antigüedad. Ejemplo de esto es el tratado La Harmónica de Ptolomeo (Siglo II d. C.). González (2014) plantea que, si bien, Ptolomeo matiza algunas ideas presentes en los tratados pitagóricos previos, este critica a Aristoxeno y a sus seguidores por poner demasiado énfasis

${ }^{6}$ González $(2014,752)$ sostiene que Aristóxeno, al dar importancia a las necesidades prácticas de hacer y ejecutar música, rechazó la relación que establecieron los pitagóricos entre la música y lo numérico. 
Numerus surdus y armonía musical. Sobre el temperamento igual y el fin del reinado pitagórico de los números Lianggi Espinoza; Juan Redmond; Pablo César Palacios Torres; Ismael Cortez Aguilera

en la práctica por sobre la teoría musical y reafirma el principio pitagórico de la imposibilidad de dividir el tono en partes iguales. La influencia paradigmática del pitagorismo de concebir a la música como ratios se ve con claridad en Ptolomeo, pues en su tratado de astronomía, el Almagesto, muestra su capacidad de encontrar valores aproximados de magnitudes inconmensurables, mas no usa estos conocimientos en su teoría musical (González 2014).

En definitiva, la importancia del pitagorismo en la antigüedad delineó la manera de concebir a la música durante la Edad Media. Y el pilar de esta influencia fue Boecio, quien en el siglo VI publicó De institutione musica. El autor fue considerado una autoridad por parte de los tratadistas musicales del Medievo, particularmente porque su obra sistematizó la tradición musical de la antigüedad desarrollada durante más de mil años. A su vez, el tratado pitagórico de teoría musical de Boecio proyectó estos saberes por mil años más en Occidente. Las categorías de Boecio de música mundana, música humana y música instrumental, determinaron que las prácticas musicales y las indagaciones en torno a ellas se centraran en la música vocal. Esto derivó en que los instrumentos se relegaran al último plano de la expresión musical y se marginaran como objeto de interés por parte de los tratadistas medievales.

Otro rasgo importante a destacar de la obra de Boecio es que, para la cristiandad, supuso un paradigma que relacionó la música con la moral. Esto conllevó en los tratados medievales un desplazamiento de los problemas teóricos musicales, lo que incidió aún más en que el tema de los inconmensurables fuese invisibilizado hasta fines de la Edad Media.

\subsection{Traducción de los Elementos de Euclides: los números sordos y sonoros}

Al ocultamiento de los pitagóricos de los inconmensurables, se suma la desaparición en Occidente de los Elementos de Euclides, una de las obras matemáticas más importantes de la antigüedad donde se trata el tema de los inconmensurables. Brigitte van Wymeersch, en un interesante artículo (2008), señala que luego de un periodo de extravío o desconocimiento, la obra de Euclides llega a Occidente en el siglo XII desde el mundo árabe, infinitamente más culto que el Occidente de los primeros diez siglos de nuestra era.

Van Wymeersch (cf. 2008) señala que, en la primera traducción registrada de los Elementos, de Gérard de Cremona (1114-1187), desde el árabe, aparece por primera vez la traducción de la palabra árabe assam (lo que actualmente entendemos como número irracional) traducida al látin como surdus. Esta traducción fue inspirada por la obra del gran matemático Al-Khwârizmî (c.825), quien considera a los números o cantidades racionales como audibles o sonoros, y a los números o cantidades irracionales como sordos, inaudibles o inexpresables. Como bien indica van Wymeersch (cf. 2008, 98), esta traducción fue considerada por algunos autores como errónea (cf. Beaujouan 1991). Dado que la palabra traducida apunta en cierto modo a la inconmensurabilidad, se pensó que lo

Revista de Humanidades de Valparaíso, 2020, No 16, 137-167

(c) (1) (9) CC BY-NC-ND 
Numerus surdus y armonía musical. Sobre el temperamento igual y el fin del reinado pitagórico de los números Lianggi Espinoza; Juan Redmond; Pablo César Palacios Torres; Ismael Cortez Aguilera

apropiado era la significación más abstracta de irracional. Sin embargo, van Wymeersch señala que la idea de números surdus encubre matices fundamentales de la concepción de número de la época y que la elección de esta palabra está lejos de ser un error. En efecto, y por el contrario, dado el contexto desde donde emerge la noción, la traducción de surdus es la más apropiada.

Plus profondément, l'irrationalité est une notion désarçonnante car elle met à mal le système de pensée dans lequel elle est apparue, à savoir la philosophie pythagoricienne. Et c'est lorsqu'on l'inscrit dans cette sphère de pensée qu'on peut comprendre la pertinence de la traduction latine numerus surdus, et la cohérence de la terminologie d'Al-Khwârizmî. (van Wymeersch 2008, 99)

De esta manera, junto con su homólogo de número sonoro, el número sordo remite a una concepción del universo y a la estrecha relación existente entre las matemáticas y la música. Y es en medio de esta relación donde se comienza a problematizar el paradigma pitagórico de la música, en el marco de la división de intervalos musicales en partes iguales y la formulación matemática del temperamento igual.

\section{Invención de los instrumentos de cuerda con trastes.}

Durante el siglo XVI, las artes mecánicas alcanzaron un desarrollo notable y nunca visto. En efecto, como señala Paolo Rossi en su libro I filosofi e le macchine 1400-1700, los primeros indicios de un nuevo enfoque del saber y de la ciencia se encuentran en las obras de los técnicos de finales del siglo XVI $(2009,22)$. Los individuos que trabajaban en talleres arsenales y tiendas de manufactura estimaron su quehacer como una forma de conocimiento. Así, llegaron a teorizar aspectos de su trabajo y a asignarle un sentido distinto al habitual de su época, en el que se le concebía como una actividad subsidiaria del saber especulativo. Y esto último no es ajeno a la música y constituye el horizonte teórico de nuestra investigación: explorar el papel protagónico que tuvieron los instrumentos de cuerda con trastes en la producción de conocimiento.

Como veremos a continuación, la discusión en torno al temperamento igual surge en el siglo XVI gracias a la valorización y desarrollo de los instrumentos de cuerda con trastes. Los instrumentos de cuerda pulsada son aquellos cuyas cuerdas se hacen vibrar con los dedos, las uñas o plectro (uñeta). Si bien, estos instrumentos tienen sus orígenes en el mediterráneo oriental, fue en el mundo árabe donde se creó el oud, antecesor del laúd occidental y sus variantes (Paniagua 2018). El oud fue un instrumento de caja con un mástil (llamado tastiera), cuerdas de número indeterminado y clavijeros, y fue usado principalmente para acompañar cantos (Figura 2). Entre los siglos VII y X encontramos fuentes que mencionan la existencia de una larga tradición de fabricantes de oudes en el mundo árabe (Paniagua 2018).

Revista de Humanidades de Valparaíso, 2020, No 16, 137-167

(c) $(1)(9)$ CC BY-NC-ND 


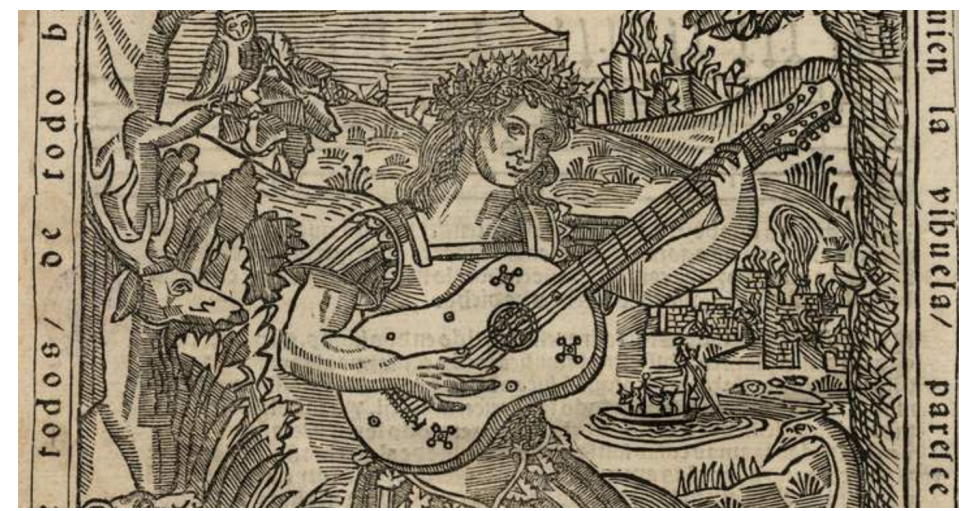

Figura 2. Imagen de Orfeo tocando la vihuela, también conocido como laúd español (de Milán 1535-1536, 10).

Respecto a la subdivisión del mástil, esta se realizaba por medio de la postura de los dedos. Paniagua plantea que el oud árabe de la Edad Media tuvo trastes de tripa que dividan el mástil. Cabe señalar que tanto las cuerdas como los trastes se solían construir con tripas de animales. Aunque también este se considera como marcas con rayas o trastes imaginarios. El traste sería el lugar o punto donde se debe posar el dedo. A su vez, se sabe que este instrumento se usó no sólo para la música práctica, sino también en la reflexión teórica de escuelas musicales árabes. Al respecto, la posibilidad de medir los sonidos a través de la subdivisión del mástil fue uno de los problemas más significativos que el instrumento heredó a la cultura occidental:

La concordancia de las cuerdas del laúd con la estructura celeste y con la orientación y el movimiento de la Tierra y su relación con las manifestaciones psicofisiológicas del hombre tanto internas (el temperamento y los humores) como externas (los colores y los perfumes) se establecieron en los primeros momentos de su existencia, cuando tenía cuatro cuerdas (Paniagua 2018, 43).

Paniagua (2018) plantea que el oud árabe, al introducirse en Occidente en torno al siglo X, se transforma en el laúd medieval. A su vez, Gómez (1992) evidencia que en muchas cartas enviadas entre los nobles españoles a fines del siglo XIV y comienzos del $\mathrm{XV}$ se hace mención de diversos tocadores de vihuela y de laúd. En este mismo sentido, las iconografías y el arte de este período muestran que los laudistas eran parte activa de la vida musical de las cortes, por lo que podemos deducir que el instrumento gozó de amplia popularidad en la Europa tardomedieval. En particular, en España, el instrumento pasó a conocerse como vihuela de mano o laúd español, donde gozó de gran prestigio y fue el instrumento preferido de las cortes españolas de los siglos XV y XVI.

En referencia a la práctica musical de esta época, la música escrita del siglo XV es eminentemente vocal y con distintas voces de manera simultánea. A comienzos del siglo 
Numerus surdus y armonía musical. Sobre el temperamento igual y el fin del reinado pitagórico de los números Lianggi Espinoza; Juan Redmond; Pablo César Palacios Torres; Ismael Cortez Aguilera

XVI, esta polifonía se había desarrollado a un punto tal que las obras escritas tuvieron de seis a ocho voces sonando al mismo tiempo. A su vez, durante el siglo XVI la música instrumental comenzó a recuperar la práctica musical de la melodía con acompañamiento, a imitación del melos de la antigüedad, en donde los instrumentos acompañaban a una única melodía cantada (Gómez 1992). Por ejemplo, en el tratado de Luis de Milán (1535-1536) se sigue escribiendo polifonías y contrapuntos para la Vihuela, mientras que en Alonso de Mudarra en 1546 ya se ve una tendencia en el uso de melodías con acompañamiento de acordes (Pope 1961). A finales del siglo, esta práctica musical fue defendida por teóricos como Vicencio Galilei y Josefo Zarlino.

La propuesta del uso de una voz acompañada en armonía con instrumentos (seconda pratica) generó un cambio radical en la forma de concebir y practicar la música en el siglo XVI. Pero a la vez, trajo una gran crisis al sistema de afinación pitagórica. En efecto, con la polifonía y el contrapunto no hubo significativos problemas de afinación, más cuando los instrumentos comenzaron a tocar acordes para acompañar a las voces, el sistema musical pitagórico entró en crisis. En este contexto, se recuperan los problemas matemáticos de la música y los problemas teóricos relativos a la medición de los sonidos, y el juicio de Boecio pierde fuerza en el ámbito de la práctica de música instrumental. Además, el clima humanista en las cortes españolas concibió a la vihuela de mano como un instrumento heredero de la lira de la antigüedad, cuya reputación incidió en que se comenzara a escribir y publicar las obras musicales para este instrumento (Pope 1961). Todos estos factores incidieron en la teorización de los sistemas de afinación de los instrumentos de cuerda con trastes y la consecuente crisis que esto detonó en el paradigma musical pitagórico de la Edad Media.

Respecto a la incorporación de trastes al laúd occidental, el tratado De inventione et usu musicae de Tinctoris (1917), publicado en 1485, señala la existencia de laudes con trastes. Plantea que las cítaras tenían una parte elevada de madera denominadas trastes, las cuales estaban dispuestas proporcionalmente en el mástil (Libro IV, iv). Y fue la ubicación de los trastes en el mástil el hecho que hizo que los problemas teóricos de afinación del instrumento comenzarán a desafiar el paradigma pitagórico de la música. En efecto, los trastes tienen la característica de que las divisiones realizadas sobre el mástil afectan de manera simultánea a todas las cuerdas del instrumento (García 2013). Este hecho derivó que los fabricantes de instrumentos (actualmente luthiers) comenzaron a dividir el mástil ubicando los trastes de manera proporcional, generando el surgimiento de la idea del temperamento igual en la teoría musical.

Es el caso de una técnica de ubicación de los trastes que usaron los fabricantes de instrumentos (Mersenne 1637, 48) y que Vincenzo Galilei describió en su tratado musical Dialogo della musica de 1581 (García 2014). Vincenzo, que era laudista, planteó que se puede dividir, en el mástil del laúd, la octava en doce partes iguales a través de una proporción geométrica de razón 17/18. El método consiste en tomar la longitud de la cuerda y tomar 17/18 partes. Posteriormente, hacer lo mismo sobre las 17/18 partes, y así 
Numerus surdus y armonía musical. Sobre el temperamento igual y el fin del reinado pitagórico de los números

Lianggi Espinoza; Juan Redmond; Pablo César Palacios Torres; Ismael Cortez Aguilera

sucesivamente. Al repetir el proceso doce veces, se llega de manera muy aproximada a la mitad de la cuerda. Es decir, se divide la cuerda aproximadamente en 12 partes iguales siguiendo una división proporcional. Este método que documenta Vincenzo era ampliamente ocupado por los fabricantes de instrumentos del siglo XVI.

Ahora bien, cuando este sistema de división del mástil se teoriza, buscando dividir exactamente la octava en doce partes iguales (es decir, proporcionales), surgen problemáticas teóricas significativas. En efecto, cada división será inconmensurable y la razón con la cual se divide la octava también será inconmensurable. Es decir, tanto las divisiones como la constante de proporcionalidad no serán ratios, sino números sordos. De esta manera, la división que funcionó de manera práctica tanto para los fabricantes de instrumentos como para los intérpretes, al ser llevada al terreno teórico, hizo surgir ideas que pusieron en crisis al paradigma musical pitagórico predominante en la época.

\section{Génesis del temperamento igual en el siglo XVI}

Como veremos a continuación, la teorización del temperamento igual puso en el centro del debate la noción de inconmensurabilidad e impulsó el desarrollo de nuestra concepción actual de los números irracionales. Después que el temperamento igual se instaura en la práctica musical de los instrumentos de cuerda con trastes, teóricos musicales, al final del siglo XVI, le dan una formulación teórica. Para explorar esto, necesitamos ir a comienzos de este siglo, donde ya se ven fracturas del sistema pitagórico en el ámbito de la teoría musical. En efecto, en diversos tratados musicales del siglo XVI, encontramos el uso del teorema de Euclides para dividir diversos intervalos musicales en partes iguales, y lo realizan en el marco de la construcción de temperamentos musicales.

\subsection{Momento 1: la división de los intervalos musicales en partes iguales}

García (2003) plantea que, en el siglo XVI, la cuerda se dividió siguiendo el sistema pitagórico de la justa entonación que surge a partir de la octava $(2 / 1)$, quinta $(3 / 2)$, cuarta $(4 / 3)$, tercera mayor (5/4) y tercera menor (6/5). Este sistema funcionaba para ciertos géneros musicales (diatónicos, como lo son la escala mayor y menor), pero tenía problemas cuando se incluían los semitonos (u otros intervalos menores al tono existente en la época). García (2003) plantea que la justa entonación, en su completitud, generaba una división de la octava en veinticuatro partes, lo cual era difícil llevar a la práctica en el caso de varios instrumentos. De aquí, surge la necesidad de la incorporación de temperamentos para disminuir esta cantidad de notas.

El temperamento consiste en la distribución de pequeños microintervalos que surgen de la justa entonación, dividiéndolos y distribuyéndolos en el resto de los intervalos musicales. De esta manera, ciertos sonidos puros son levemente aumentados o disminuidos en una proporción que sea tolerable al oído (García 2003). Por ejemplo, bajo el sistema

Revista de Humanidades de Valparaíso, 2020, No 16, 137-167

(c) $(1)(9)$ CC BY-NC-ND 
de la justa entonación, los intervalos bemoles son distintos a los sostenidos. Al temperar, por decirlo de algún modo, se aumenta el bemol y se disminuye el sostenido, de manera que en vez de usar en el teclado dos teclas distintas para bemoles y sostenidos se utilice solo una (como son los pianos y teclados modernos), reduciendo las notas en la que se divide la octava (Figura 3) ${ }^{7}$.

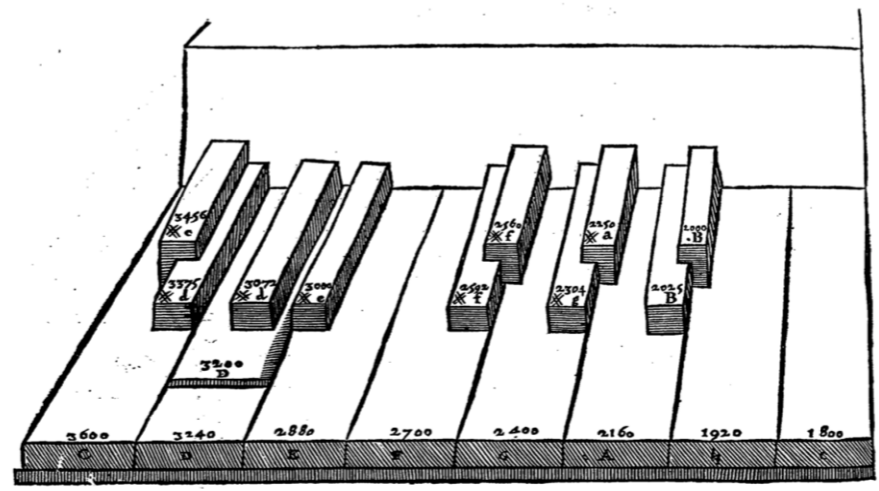

Figura 3. Teclado armónico en una división de la octava en 19 partes (las notas bemoles son diferentes a las sostenidas), en Mersenne (1637, 362).

Fue justamente en la construcción de tales temperamentos que los teóricos musicales del siglo XVI comenzaron a utilizar el teorema de Euclides para dividir ciertos intervalos musicales en partes iguales ${ }^{8}$. Estos autores sostuvieron que si bien, era imposible aritméticamente encontrar la mitad de varios intervalos, esto si era posible por medio de métodos geométricos. Está el ejemplo de Faber Stapulensis (1496) quien usó el teorema de Euclides para dividir las consonancias bf, be, bd y bc en partes iguales, obteniendo respectivamente las medias proporcionales bk, bi, bh y bg (Figura 4).

\footnotetext{
${ }^{7}$ Existen distintas formas de explicar el temperamento. En este escrito, seguimos la explicación que García (2003) desarrolla a partir de las obras de Salinas y Zarlino, que los autores analizados en esta investigación. ${ }^{8}$ González (2014) sostiene que los teóricos musicales de la antigüedad no utilizaron métodos geométricos para dividir intervalos musicales en partes iguales.
} 
Numerus surdus y armonía musical. Sobre el temperamento igual y el fin del reinado pitagórico de los números Lianggi Espinoza; Juan Redmond; Pablo César Palacios Torres; Ismael Cortez Aguilera

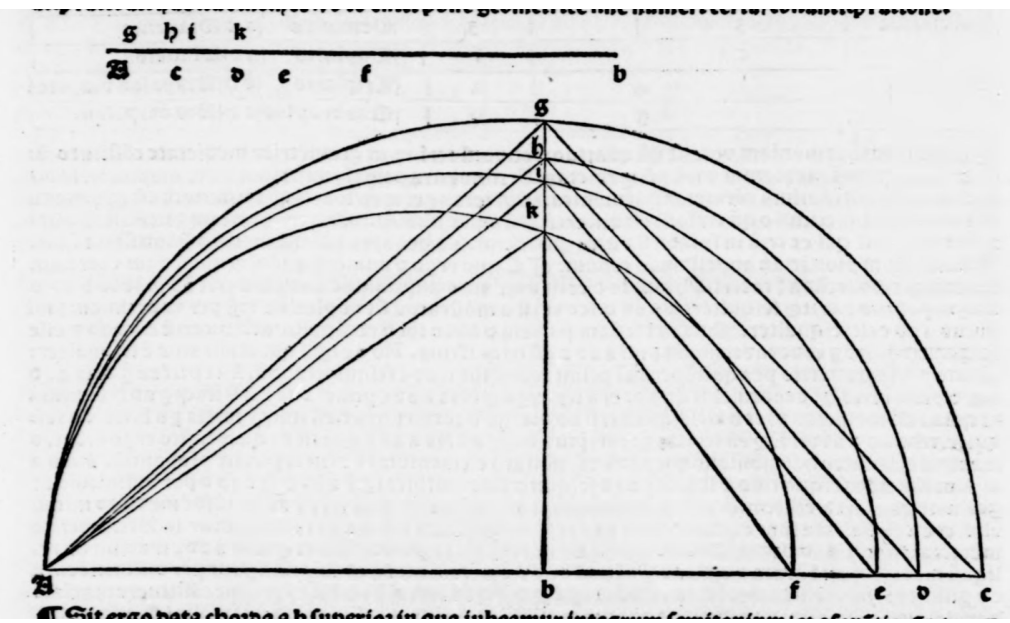

Figura 4. Uso del teorema de Euclides para dividir consonancias en Arithmetica et musica de Faber Stapulensis $(1496,111)$.

A su vez, algunos teóricos musicales usaron la división geométrica de intervalos en partes iguales para la elaboración de diversos temperamentos. Es el caso de Gioseffo Zarlino que en su Istitutioni harmoniche (1558), se refiere a la posibilidad de dividir geométricamente un tono en dos partes iguales mediante el uso del teorema de Euclides ${ }^{9}$ (Figura 5):

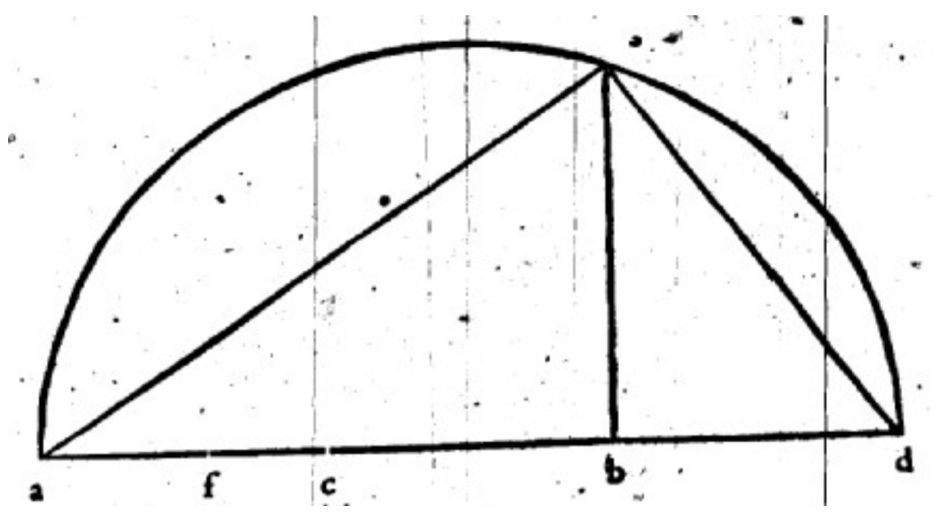

Figura 5. Uso del teorema de Euclides para dividir consonancias en Istitutioni harmoniche de Zarlino $(1558,94)$.

Zarlino también explica una técnica para dividir un intervalo en tres o más partes iguales para la construcción de otros temperamentos. Zarlino plantea que estas divisiones se pueden realizar mediante el uso del Mesolabio, un instrumento que señala creó Eratóstenes para resolver el problema de la duplicación del cubo

${ }^{9}$ Zarlino realiza esta división para construir el temperamento mesotónico de 1/4 de coma (García 2003).

Revista de Humanidades de Valparaíso, 2020, No 16, 137-167 
Zarlino también explica una técnica para dividir un intervalo en tres o más partes iguales para la construcción de otros temperamentos ${ }^{10}$. Plantea que estas divisiones se pueden realizar utilizando el mesolabio, un instrumento geométrico-mecánico que señala creó Eratóstenes para resolver el problema de la duplicación del cubo ${ }^{11}$. Tal problema consiste en encontrar un cubo cuyo volumen sea el doble de otro cubo dado. Hipócrates de Quíos (siglo V a. C.), postuló que el problema de la duplicación del cubo se puede resolver encontrando dos medias proporcionales entre una magnitud y su mitad (Barrios y Prieto 1992). En el siglo XVI, Zarlino plantea que el mesolabio de Eratóstenes permite encontrar tantas medias proporcionales que se desee. Trece años después, Zarlino vuelve a plantear estas ideas en su Dimonstrationi harmoniche (1571), En las proposiciones 9, 10 y 11 del libro 2, plantea que numéricamente no se puede dividir el tono (9/8) en dos semitonos iguales, que esta división si puede realizarse mediante el uso de la geometría y que se puede dividir cualquier cuerda en las partes iguales que se desee mediante el uso del mesolabio (Figura 6).

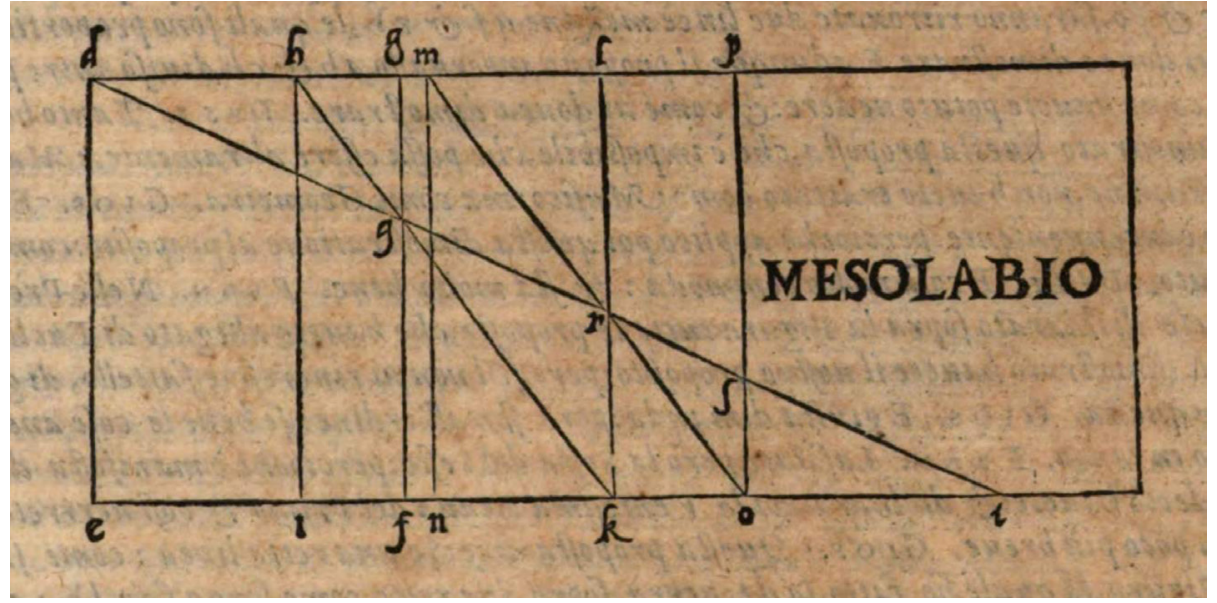

Figura 6: Ilustración del mesolabio, en Dimonstrationi harmoniche de Zarlino $(1571,163)$.

Seis años después, el español Francisco de Salinas, en su obra De música libri septem (1577), usa el teorema de Euclides para dividir algunos intervalos en partes iguales, en el contexto de la construcción de ciertos temperamentos ${ }^{12}$. En la figura 7, Salinas usa el teorema de Euclides para dividir los intervalos musicales de 5/4 y 80/81 ${ }^{13}$. En ambas construcciones Salinas usa el teorema de Euclides para construir la media proporcional

\footnotetext{
${ }^{10}$ Específicamente, los temperamentos mesotónicos de 1/3 de coma y de 2/7 de coma (García 2003).

${ }^{11}$ Para explorar el funcionamiento del mesolabio ver García (2002).

${ }^{12}$ Como por ejemplo el temperamento mesotónico de $1 / 4$ de coma (García 2002)

${ }^{13} 5 / 4$ es la tercera mayor y 80/81 es la coma sintónica, que era la diferencia entre los dos tipos de tonos que existían en la época. Con esto último logra construir un intervalo único para definir un tono.
} 
entre BA y BC, obteniendo las medias proporcionales BD. Después las copia mediante el uso del compás sobre la base de la semicircunferencia $(B D=B F)$ obteniendo de esta manera las proporciones $\mathrm{BE} / \mathrm{BF}=\mathrm{BF} / \mathrm{BA}$.
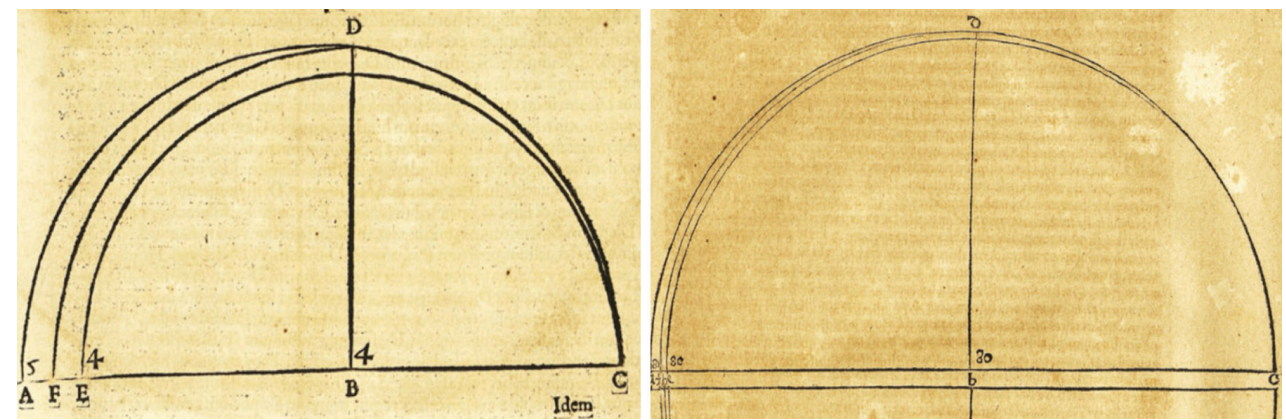

Figura 7: Uso del teorema de Euclides para dividir en partes iguales los intervalos musicales tercera mayor y coma sintónica, en De música libri septem de Salinas $(1577,158-159)$.

De esta manera, desde comienzos del siglo XVI emerge una ruptura del paradigma pitagórico en la teoría musical, con la incorporación la división de intervalos musicales en partes iguales. Al ser inconmensurables, a estas divisiones no le corresponden ratios. Este primer momento de crisis en el siglo se dio en el marco de la elaboración de temperamentos, los cuales se desarrollaron dado el reavivamiento de la música instrumental.

\subsection{Momento 2: formulación teórica del temperamento igual}

García (2003) plantea que, para los instrumentos de teclas, los teóricos musicales del siglo XVI crearon temperamentos de modo de igualar los tonos, pero mantuvieron los dos semitonos donde los intervalos sostenidos son diferentes a los intervalos bemoles (Figura 3). Sin embargo, para el caso de los instrumentos con trastes, dado que los trastes afectan de manera simultánea a todas las cuerdas, propusieron un temperamento que igualara todos los semitonos. Este es el temperamento igual, que divide la octava en doce partes iguales.

García (2014) sostiene que el español Francisco de Salinas fue el primer teórico en describir con precisión matemática el temperamento igual. El formuló teóricamente este temperamento y recomendó su uso en instrumentos de cuerdas con trastes. Cabe señalar que la división que se hace de la octava con este temperamento es una alteración de todos los intervalos pitagóricos, de modo que ningún intervalo queda justo o puro. Es decir, si bien, los otros temperamentos ya manifestaban pequeñas rupturas con el paradigma mu- 
sical pitagórico, este temperamento lo desafía completamente ${ }^{14}$. En efecto, en este temperamento ningún intervalo musical corresponde a alguna ratio, y en el tiempo de Salinas solo es posible construirlo y teorizarlo geométricamente.

Usando el teorema de Euclides no se puede dividir la octava en doce partes iguales. En efecto, aplicando iteradamente este teorema se pueden encontrar solo 2, 4, 8 o 16 partes iguales (u otras potencias de 2). Por este motivo, para dividir la octava en 12 partes iguales, se necesita incluir un método que permita dividir un intervalo en tres o más partes iguales. Salinas plantea que esto se puede hacer mediante el uso del mesolabio. No explica en detalle el proceso de construcción, pues señala que esto ya se había explicado en la Arquitectura de Vitrubio así como en otros tratados antiguos. Sin embargo, al postularlo, es el primer teórico que publica una formulación teórica del temperamento igual (figura 8). Y no solo eso, sino que también es consciente de la imposibilidad de hacerlo de forma numérica por la respectiva inconmensurabilidad y defiende su pertinente uso en la música para instrumentos de cuerda con trastes (García 2014).

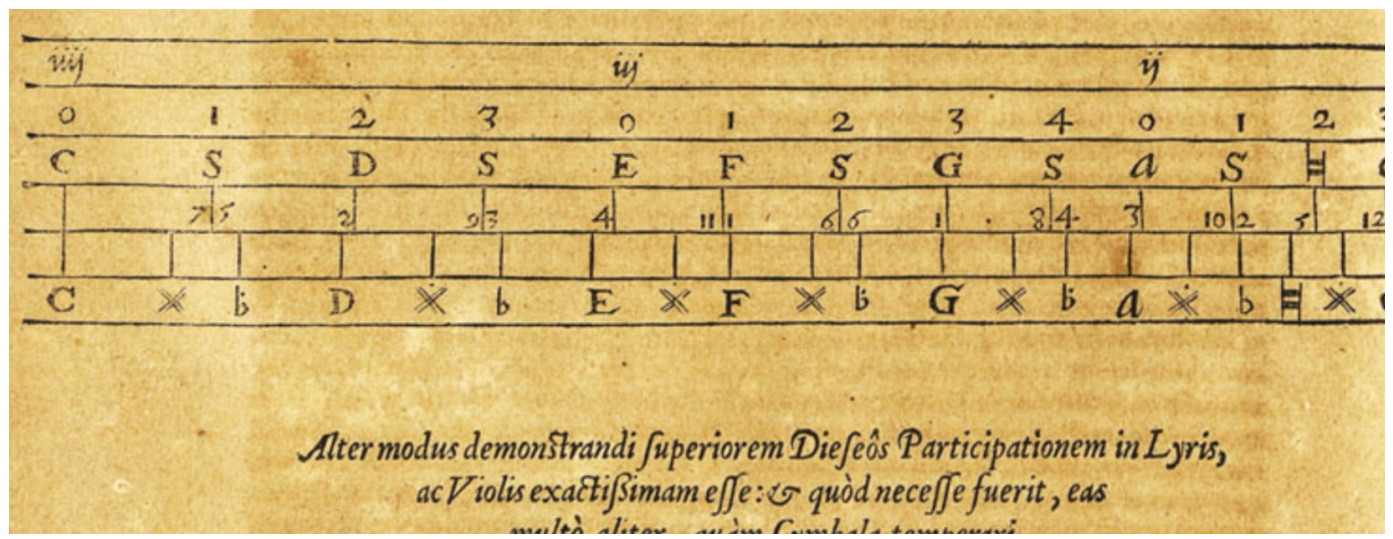

Figura 8. Imagen de De música libri septem de Salinas, en la que se compara el temperamento igual con un temperamento mesotónico, en el cual las notas bemoles son distintas a las sostenidas (Salinas 1577, 170).

Once años después, en su Sopplimenti musicali (1588), Zarlino desarrolla exhaustivamente la formulación teórica del temperamento igual. En la proposición XXVII del Libro IV, Zarlino explica que esta forma de distribuir en 12 partes iguales se aplica para los instrumentos de cuerda con trastes, como lo son el Laúd y la Viola, acompañando una extensa explicación respecto al contexto de esta división. Después, en las proposiciones

\footnotetext{
${ }^{14}$ Hubo detractores al uso del temperamento igual en la música. En efecto, dado que la tendencia estética del barroco fue acentuar las consonancias y disonancias, estos consideraron que si se atenuaban las disonancias, la música perdería efectividad para mover los afectos.
} 
XXVIII y XXVIX, presenta una explicación detallada del método de división del mástil usando iteradamente la razón 17/18 (Figura 9). Zarlino calcula estas razones y concluye que con este método no se logra dividir exactamente la octava en doce partes iguales.

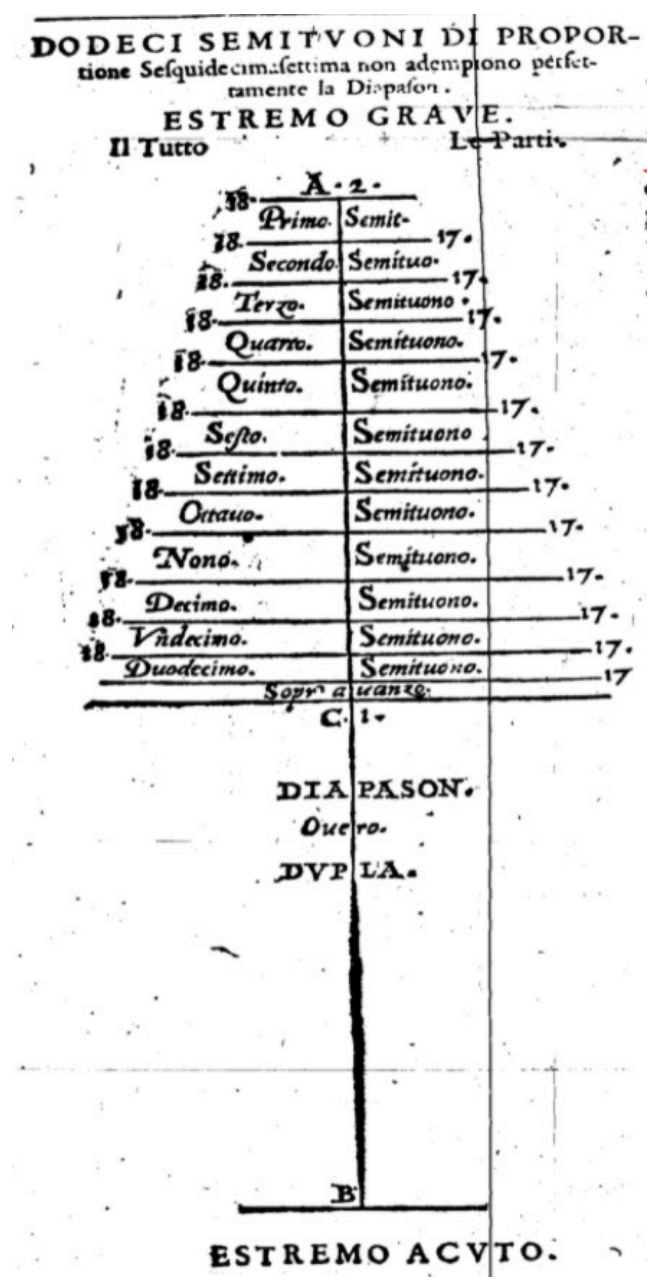

Figura 9. Imagen de Sopplimenti musicali de Zarlino en la que analiza el método de división del mástil usado por los fabricantes de instrumentos, siguiendo la razón 17/18 (Zarlino 1588, 202).

A su vez, Zarlino explica en la proposición XXX del libro IV el uso del mesolabio para dividir el mástil en 12 partes iguales, acompañando de la ilustración de la figura 10. 


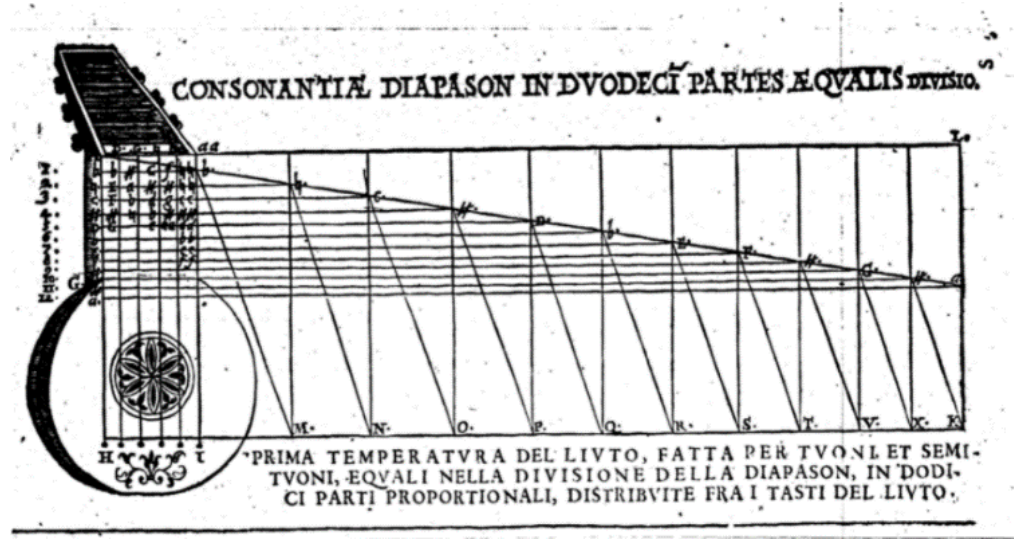

Figura 10. Imagen de Sopplimenti musicali de Zarlino, en la que explica la división del mástil en doce partes iguales mediante el uso del mesolabio (Zarlino 1588, 209).

Además, este teórico musical explica en la proposición 20 del libro IV una técnica geométrica-mecánica para encontrar dos medias proporcionales, el cual atribuye a Filón de Bizancio (280-220 a. C.). El autor destaca la simplicidad de la construcción y su gran aplicabilidad para resolver el problema de la división del mástil en 12 partes iguales (capítulo 20, Libro VI). El método consiste en ubicar en $a b$ la cuerda y en $b c$ su mitad, es decir, su octava, construyendo el rectángulo abcg (Figura 11). Después, traza la diagonal $c a$ y construye sobre ella el círculo que pasa por $b$ y $g$. Posteriormente, prolonga la recta $g c$ y toma un punto $d$ sobre esta última recta y traza la recta $d b$, obteniendo el punto $f$ sobre la recta prolongada de ag. Finalmente, mueve mecánicamente el punto $d$ de modo que la distancia $d b$ sea igual a $f e$. Cuando ocurra esto, también se tendrá que la distancia $d h$ será igual a $h f$. En definitiva, tendrá las rectas $f a$ y $d c$, las dos medias proporcionales entre la cuerda y su octava. Esta construcción también es válida para otros intervalos musicales ${ }^{15}$.

${ }^{15}$ La demostración geométrica de esta proposición se puede consultar en García $(2003,106)$. 
Numerus surdus y armonía musical. Sobre el temperamento igual y el fin del reinado pitagórico de los números Lianggi Espinoza; Juan Redmond; Pablo César Palacios Torres; Ismael Cortez Aguilera

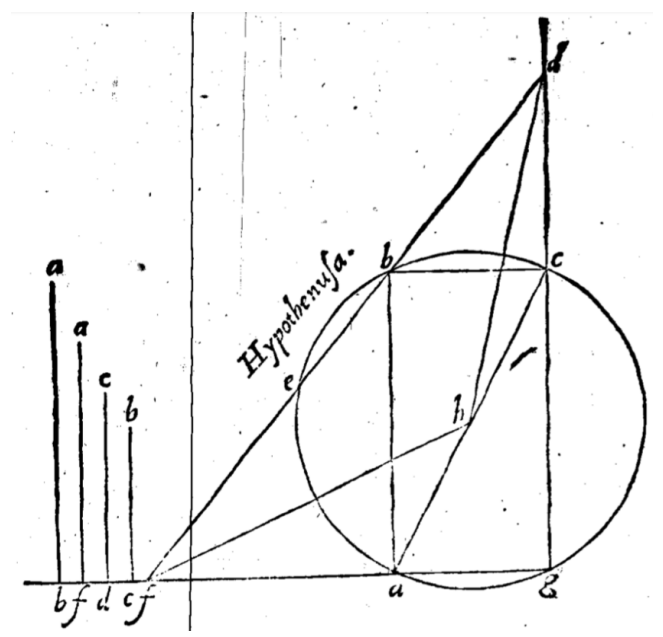

Figura 11. Imagen de Sopplimenti musicali en la que Zarlino presenta la construcción geométrica de dos medias proporcionales, atribuida a Filón de Bizancio (Zarlino 1588,182$)$.

Posteriormente, en la proposición 31, presenta una división del mástil en 12 partes iguales mediante construcciones geométricas (Figura 12). Primero, usa el teorema de Euclides para encontrar una media proporcional entre la cuerda y su octava. Después, usa nuevamente el teorema de Euclides sobre los dos intervalos encontrados dividiendo el mástil en cuatro partes iguales. Por último, usa la construcción geométrica-mecánica de Filón de Bizancio para dividir cada uno de los cuatro intervalos resultantes en tres partes iguales ${ }^{16}$, consiguiendo una división del mástil en doce partes iguales. Esto lo postula para su uso en instrumentos de cuerda con trastes, como se puede ver en la imagen, en la que incluye el instrumento musical dentro de la construcción geométrica.

\footnotetext{
${ }^{16}$ En efecto, así como con una media proporcional se divide el mástil en dos partes iguales, con dos medias proporcionales se divide en tres partes iguales.
} 
Numerus surdus y armonía musical. Sobre el temperamento igual y el fin del reinado pitagórico de los números Lianggi Espinoza; Juan Redmond; Pablo César Palacios Torres; Ismael Cortez Aguilera

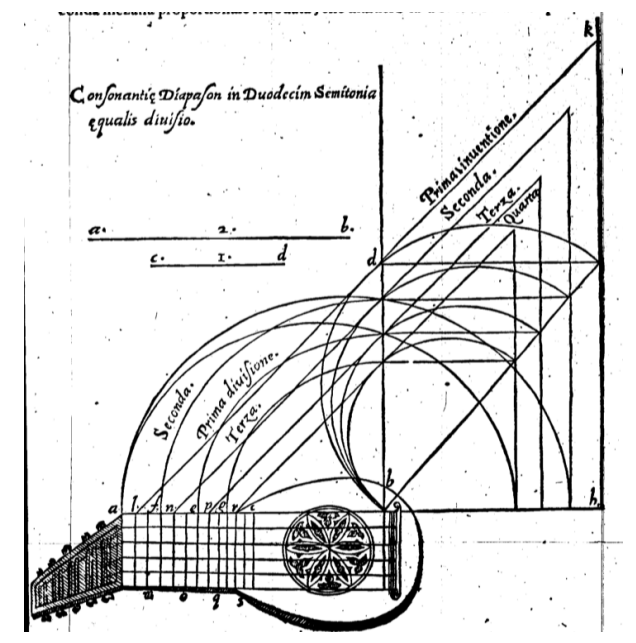

Figura 12. Imagen de Sopplimenti musicali en la que Zarlino explica el uso del teorema de Euclides y la construcción geométrica de Filón de Bizancio para la división del mástil en doce partes iguales (Zarlino 1588, 211).

Un aspecto interesante es que, tanto en el método con el uso del mesolabio, como en las construcciones usando el teorema de Euclides y el método de Filón de Bizancio, Zarlino subraya que logra dividir la octava en doce divisiones iguales sin que falte o sobre algún espacio por mínimo que sea (García 2014). Es decir, explica los métodos geométricos mediante los cuales se construye con exactitud el temperamento igual, sin el error obtenido con el método de los fabricantes de instrumentos. De esta manera, se plantea una teorización completa del temperamento igual, en el contexto geométrico, y recomendada para ser aplicada en instrumentos de cuerda con trastes.

De esta manera, Salinas y Zarlino desarrollan la división geométrica del mástil en doce partes iguales para instrumentos de cuerda con trastes. Con tal división plantean una ruptura radical con la concepción pitagórica de la música mediante ratios. Estos autores concuerdan con Boecio en el hecho de que la división en partes iguales no puede hacerse numéricamente, mas sostienen que se puede realizar de manera geométrica. En este contexto, dado que el temperamento igual propone una división del mástil en que cada sonido es una magnitud inconmensurable, desafía radicalmente el paradigma musical pitagórico. En efecto, a ninguna división de la cuerda le corresponde una ratio. Cabe señalar además que Salinas y Zarlino proponen este temperamento solo para instrumentos de cuerda con trastes. Para otros instrumentos, como el clavecín (instrumento de teclas), proponen otros temperamentos en el que mantienen algunas consonancias puras. 
Numerus surdus y armonía musical. Sobre el temperamento igual y el fin del reinado pitagórico de los números Lianggi Espinoza; Juan Redmond; Pablo César Palacios Torres; Ismael Cortez Aguilera

\subsection{Momento 3: la aritmetización del problema}

Un último hito importante en el problema del temperamento igual en el siglo XVI tiene relación con su aritmetización. El neerlandés Simón Stevin ${ }^{17}$ publicó en 1585 su Aritmética. En esta obra, después de cuestionar la estrechez de la noción de número usada en su época, derivada de la concepción de Euclides, propone concebir al número como una cantidad continua, conceptualizando al número irracional como un número más entre los naturales y los racionales (Crone et al. 1961). A su vez, propone una notación decimal para las razones, define los números geométricos, las potencias y las raíces, y desarrolla técnicas aritméticas para sus cálculos numéricos.

En el problema XLV de su libro plantea que, dados dos números cualesquiera, se pueden encontrar entre ellos las medias proporcionales que se deseen. Para hacer esto, articula las técnicas geométricas usadas en la época con sus métodos aritméticos ${ }^{18}$. Por ejemplo, dado los números 2 y 10, y siguiendo el teorema de Euclides, sostiene que su media proporcional será la raíz cuadrada de su producto, es decir, . Asimismo, presenta técnicas aritméticas para encontrar dos y cuatro medias proporcionales, y propone el uso iterado y combinado de las técnicas para encontrar números superiores de medias proporcionales entre dos números dados. En esencia, en su aritmetización propone las mismas iteraciones que Zarlino realiza en su método geométrico-mecánico para la construcción del temperamento igual (Figura 12).

También, en la sección del tratado que hace referencia a los inconmensurables, Stevin desarrolla diversas técnicas para el cálculo de tales magnitudes mediante el uso del teorema de Euclides para raíces cuadradas. Para las cúbicas, menciona la existencia de una diversa cantidad de métodos geométricos-mecánicos para la construcción de dos medias proporcionales, entre los cuales menciona el método de Filón de Bizancio. Para el caso de raíces superiores, señala que se pueden obtener mediante el uso del "instrumento de Eratóstenes", que interpretamos se refiere al mesolabio.

Respecto a la relación explícita de estas técnicas aritméticas con el problema del temperamento igual, en su Aritmética, Stevin $(1585,57)$ hace una alusión directa a la teoría musical. A su vez, en unos apuntes de un tratado de teoría musical, Vande Spiegheling der Singconst (Crone et al. 1966), Stevin usa las técnicas aritméticas descritas previamente para el cálculo del temperamento igual, mediante el cálculo de raíces (Figura 13). Estos apuntes están datados también en 1585, y son parte de una obra que finalmente no publicó. Dada su falta de expertise en teoría musical, actualmente existe un debate respecto de si realmente en estos apuntes formuló el temperamento igual (Floris 1987; Rasch 2008). Sin embargo, es indudable que Stevin formuló los métodos matemáticos para resolver

\footnotetext{
${ }^{17}$ Hay una resolución en China en los mismos años, de Chu Tsai-Yü en los mismos años.

${ }^{18}$ Cabe señalar que junto los métodos aritméticos surgieron nuevas notaciones, como la de raíz y la exponencial.
}

Revista de Humanidades de Valparaíso, 2020, No 16, 137-167 
aritméticamente el problema de este temperamento. En la figura 13 se presenta las secciones de este apunte, recuperado y escrito en simbología matemática del siglo XIX, de la formulación matemática y resultados numéricos que Stevin provee al temperamento igual.

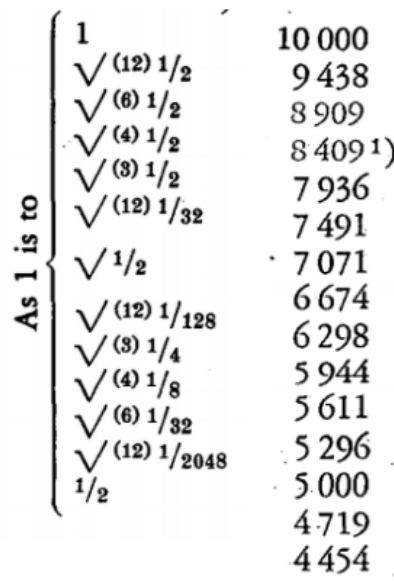

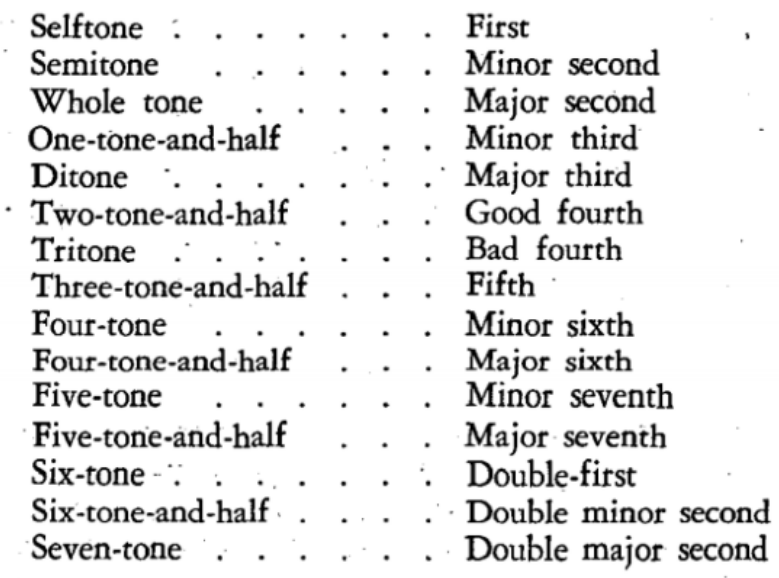

Figura 13. División aritmética de la octava en 12 partes iguales en Vande Spiegheling der Singconst de Stevin (Crone et al. 1966, 443).

Cabe señalar que tanto en su Aritmética, como en su Vande Spiegheling der Singconst, Stevin plantea una crítica directa a la concepción de los números irracionales de su época y del paradigma numérico de los griegos. En ambas obras plantea un cuestionamiento a la concepción, que atribuye a los griegos, de considerar a los inconmensurables como magnitudes irracionales, inexplicables o inapropiadas para ser consideradas números. Al respecto, plantea categóricamente que no existen números irracionales, absurdos, inexplicables o sordos, en el sentido de que inconmensurabilidad en aritmética no implica algo fuera de la razón, sin sentido o que no se puedan explicar.

De esta manera, la aritmetización del problema desafió la dicotomía de concebir a las

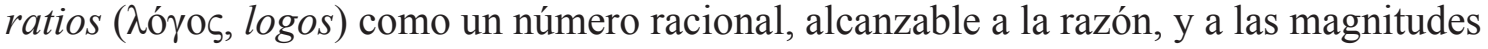
inconmensurables como no racionales, es decir, irracionales. En el terreno de la música, esta idea tiene implicancias significativas. En efecto, desafía la concepción de entender la música desde los números sonoros y sordos, así como la hipótesis pitagórica de vincular los sonidos a las ratios. Esto último genera una crisis filosófica al interior del paradigma pitagórico. En efecto, como señalamos anteriormente, la mera consideración de sonidos sin ratios derrumba uno de los pilares metafísicos del pitagorismo: la naturaleza numérica de la realidad. Y esto, a su vez, disuelve el poder integrador del mensaje pitagórico que se proponía integrar distintos saberes en una unidad.

En definitiva, la Aritmética de Stevin no solo cambia la concepción de número en Occidente, sino que también desarrolla las técnicas aritméticas necesarias para resolver 
Numerus surdus y armonía musical. Sobre el temperamento igual y el fin del reinado pitagórico de los números Lianggi Espinoza; Juan Redmond; Pablo César Palacios Torres; Ismael Cortez Aguilera

el problema del temperamento igual. En efecto, tales técnicas subyacen a cálculos numéricos de este temperamento realizados en el siglo XVII. Es el caso de Marin Mersenne, quien, en su Harmonie Universelle (1637), brinda aproximaciones por exceso y por defecto a la división del temperamento igual, obteniendo un error que considera imperceptible al oído humano (Figura 14).

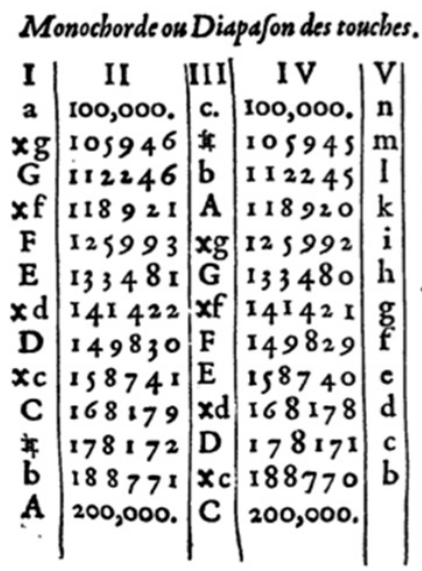

Figura 14. Valores aproximados por exceso y por defecto de la división de la octava en doce partes iguales, en Mersenne $(1637,38)$.

Así se resuelve por completo el problema de la teorización matemática del temperamento igual. Ahora bien, aún faltan tres siglos para que este sistema de afinación se asuma como el estándar para todos los instrumentos occidentales. Desde la irrupción de la melodía acompañada y la música instrumental en el siglo XVI, este temperamento comenzó a tener una gravitación mayor en el quehacer musical. Sin embargo, como plantea Katharine Ellis (2001), es durante el siglo XIX cuando se instaura el temperamento igual como canon de la música occidental. Según la autora, este proceso fue impulsado particularmente por la práctica del piano en las casas, el gusto por la ópera, la comercialización de repertorios musicales a través de partituras y la adopción de los instrumentos temperados como la guitarra en las músicas folclóricas.

Sin duda esta estandarización ofreció una solución a la problemática de hacer música con diversos instrumentos de manera simultánea y propició una circulación más amplia y rápida de los repertorios musicales. Sin embargo, también impuso al temperamento igual como norma de valor estético naturalizado, donde lo perfecto, lo audible y lo estéticamente correcto está contenido solamente en esta proporción matemática. Una consecuencia de esta estandarización puede ser el hecho de soslayar las sonoridades propias de culturas ancestrales y la de otros temperamentos que tuvieron gran importancia para las sociedades en diferentes momentos de la historia. En esta línea, algunos compositores contemporáneos, en su incansable búsqueda de nuevas sonoridades, han vuelto a explorar otros temperamentos, como lo es el sistema microtonal. Pero más allá de esto, la estan- 
Numerus surdus y armonía musical. Sobre el temperamento igual y el fin del reinado pitagórico de los números Lianggi Espinoza; Juan Redmond; Pablo César Palacios Torres; Ismael Cortez Aguilera

darización del temperamento igual ha delineado la manera moderna y contemporánea de concebir la música y ha propiciado el enorme desarrollo musical que hemos visto durante el último siglo en el mundo.

\section{Conclusión}

En este artículo nos propusimos mostrar el rol determinante que tuvieron en el siglo XVI los instrumentos de cuerda con trastes en el desmoronamiento de uno de los enfoques más influyentes en la historia de la filosofía: el pitagorismo. Para esto, primero describimos elementos del sistema unificador pitagórico y su relación con la teoría musical, mediante la identificación de los sonidos puros con ratios. Nos referimos a la evitación de los pitagóricos de las magnitudes inconmensurables, tanto en su perspectiva filosófica como musical. En esta línea, detallamos el postulado pitagórico respecto a la imposibilidad de la división del tono en partes iguales. Después, hicimos referencia a la influencia del pitagorismo como paradigma predominante de la teoría musical en occidental durante la Edad Media, así como la consecuente invisibilización de los inconmensurables durante este periodo. Nos referimos a la traducción de los Elementos de Euclides desde el mundo árabe, y al dilema respecto a la traducción de las magnitudes inconmensurables como números sordos (Van Wymeersch 2008).

Posteriormente, como punto fundamental de nuestra argumentación, nos referimos a algunos hitos históricos relevantes de la invención, la fabricación y el uso de los instrumentos de cuerda con trastes en Occidente. Hicimos alusión a los cambios que ocurrieron en el siglo XVI en el ámbito de la práctica del laúd y la vihuela, como el comienzo de la escritura musical y el paso de la polifonía a la melodía acompañada con instrumento. En paralelo, nos referimos a la incorporación de trastes a los mástiles de instrumentos con cuerda, y la progresiva afinación proporcional de los tales con la que se comenzaron a afinar estos instrumentos. Explicamos que esto fue algo característico y exclusivo de los instrumentos de cuerda con trastes, causado por las características morfológicas del instrumento, donde cada traste afecta de manera simultánea a todas las cuerdas (García 2014). Tales afinaciones proporcionales se realizaron siguiendo la técnica que usaron los fabricantes de instrumentos, descrita por Vincenzo Galilei, en la que se divide el mástil usando una progresión geométrica de razón 17/18.

Finalmente, explicamos cómo durante el siglo XVI la formulación teórica del temperamento igual para los instrumentos de cuerda con trastes provocó una gran crisis en el paradigma musical pitagórico, por la incorporación de magnitudes inconmensurables a la división de la cuerda. Dado que el paradigma pitagórico se impuso en Occidente por alrededor de dos mil años, en coexistencia con las magnitudes inconmensurables, nos hace considerar que la existencia de tales magnitudes no parece haber puesto en cuestión al paradigma. En efecto, su tesis no parece ser que a cada número debe corresponder una realidad, sino que la realidad es de naturaleza numérica, por tanto, allí donde hay realidad

Revista de Humanidades de Valparaíso, 2020, No 16, 137-167

(c) $(1)(9)$ CC BY-NC-ND 
Numerus surdus y armonía musical. Sobre el temperamento igual y el fin del reinado pitagórico de los números Lianggi Espinoza; Juan Redmond; Pablo César Palacios Torres; Ismael Cortez Aguilera

(sobre todo material) debe haber una ratio que le corresponda. Al respecto, la gran ruptura se da en términos de asumir la existencia concreta y palpable (audible) de sonidos a los que no les corresponde ninguna ratio. Y esto es exactamente lo que ocurrió durante el siglo XVI. Este socavamiento del pitagorismo lo sintetizamos en tres momentos.

En el primer momento encontramos la revalorización de la música instrumental en Occidente, motivo por el cual los teóricos musicales, ante las dificultades que proveyó el sistema pitagórico (afinación justa), comenzaron a integrar temperamentos. Al temperar, estos teóricos comenzaron a utilizar el teorema de Euclides para dividir ciertos intervalos de manera geométrica en partes iguales, divisiones que eran inconmensurables. Posteriormente, también se usó el mesolabio para encontrar dos medias proporcionales. En este primer momento, fueron las necesidades prácticas de simplificar la construcción de instrumentos lo que conlleva a esta incorporación de ciertas divisiones irracionales. Y esto puso el cimiento para el siguiente momento, en el que Salinas y Zarlino formulan la teorización matemática del temperamento igual.

En este segundo momento, toda la división de la octava son magnitudes inconmensurables, así como también la razón con la cual esta división proporcional se construye. Es decir, el temperamento igual fue, por así decirlo, la antítesis de la máxima pitagórica de los números sonoros, en el sentido que los sonidos deben corresponder a ratios. De esta manera, se introduce a la teoría musical una idea que tiene el potencial de socavar al paradigma musical que fue significativamente influyente en la antigüedad y predominante durante todo el Medioevo occidental: el pitagorismo. Finalmente, en un tercer momento, explicamos cómo la aritmetización del problema del temperamento igual en la obra de Stevin desafía la concepción de número de los antiguos, al considerar a los inconmensurables como cantidades alcanzables a la exploración racional. De esto se deduce la imposibilidad de la existencia de números "sordos" en el sentido pitagórico. De esta manera, hemos logrado nuestro objetivo de investigación.

Para terminar, cabe señalar que lo expuesto hasta aquí no significa la desaparición de la influencia pitagórica sobre la música. Efectivamente, en el temperamento igual, las ratios pitagóricas siguen siendo los principios orientadores de la división de la octava. Sin embargo, en este temperamento tales ratios comienzan a ser concebidas progresivamente desde una perspectiva más vinculada con la práctica que con la especulación. Así, fue en el terreno de la práctica, con los instrumentos de cuerda con trastes, donde comenzó el desmoronamiento del pitagorismo como sistema filosófico para la música. Y esto ha tenido significativas repercusiones hasta nuestros días. En efecto, toda la música que hoy escuchamos y los instrumentos que tocamos están fundados en este sistema de afinación. Por tanto, cuando vemos los trastes del mástil de una guitarra, estamos contemplando una síntesis de un desarrollo histórico, el cual no solo ha definido la manera actual de concebir la música y gestó la formulación de ideas matemáticas que son fundamentales en el presente, como la de número irracional, sino que incidió en el socavamiento del pitagorismo, uno de los sistemas filosóficos más influyentes en la historia. 
Numerus surdus y armonía musical. Sobre el temperamento igual y el fin del reinado pitagórico de los números Lianggi Espinoza; Juan Redmond; Pablo César Palacios Torres; Ismael Cortez Aguilera

\section{Referencias bibliográficas}

Aristóteles (1994). Metafísica. Madrid: Gredos.

Barker, Andrew (2014). Pythagorean harmonics. En Carl A. Huffman (ed.), A History of Pythagoreanism, pp. 185-203. Cambridge: Cambridge University Press.

Barrios, José, Prieto, Ramón (1992). Los problemas especiales: la duplicación del cubo I (De Hipócrates a Eratóstenes). En M. Hernández y J. Montesinos (eds.), Actas del I Seminario Orotava de Historia de la Ciencia, pp. 371-391. Canarias: Ediciones Educativas Canarias.

Beaujouan, Guy (1991). Le vocabulaire scientifique du latin medieval. En Guy Beaujouan (ed.), Par raison de nombres. L'art du calcul et les savoirs scientifiques médiévaux, VIII, pp. 346-347. Hampshire: Variorum.

Brunschvicg, Léon (1947). Les étapes de la philosophie mathématique. Paris: PUF.

Burkert, Walter (1972). Lore and Science in Ancient Pythagoreanism. Cambridge, MA: Harvard University Press.

Burnet, John (1928). Greek Philosophy, Part I, Thales to Plato. London: Macmillian and Co., Limited St. Martin's street.

Crone, Ernst, et allia (1961). Introduction. En En Ernst Crone, E. J. Dijksterhuis, R. J. Forbes, M. G. J. Minnaert, A. Pannekoek (eds.), The principal works of Simon Stevin, Vol. 2b: Mathematics, pp. 459-476. Amsterdam: C. V. Swets \& Zeitlinger.

Crone, Ernst, et allia (1966). Music. En Ernst Crone, E. J. Dijksterhuis, R. J. Forbes, M. G. J. Minnaert, A. Pannekoek (eds.), The principal works of Simon Stevin, Vol. 5: Engineering Music - Civic Life, pp. 413-464. Amsterdam: C. V. Swets \& Zeitlinger.

De Milán, Luis (1535-1536). Libro de música de vihuela de mano intitulado "El Maestro". Valencia: Francisco Díaz Romano.

Ellis, Katharine (2001). The structures of musical life. En The Cambridge History of Ninteenth-Century Music, pp. 343-370. Cambridge: Cambridge University Press.

Euclides (1774). Los seis primeros libros y el undécimo, y duodécimo de los Elementos de Euclides (Trad. Simson, R.). Madrid: D. Joachin Ibarra, Impresor de Cámara de S.M.

Faber Stapulensis, Jacobus (1496). Arithmetica et musica. Paris: Joannes Higmanus et Volgangus Hopilius.

Floris, Hendrik (1987). Simon Stevin's equal division of the octave. Annals of Science, 44(5), 471-488. https://doi.org/10.1080/00033798700200311

Galilei, Vincenzo, d. (1589). Discorso Intorno Alle Opere Di Gioseffo Zarlino: Et Altri Importanti Particolari Attenenti Alla Musica. Venezia: Appresso Giorgio Marescotti.

García Pérez, Amaya (2003). El número sonoro: la matemática en las teorías armónica de Salinas y Zarlino. Salamanca: Caja Duero.

García Pérez, Amaya (2002). El temperamento en las teorías musicales de Salinas y Zarlino uso y aplicación del “mesolabio". Revista de Musicología, 25(2), 347-361.

Revista de Humanidades de Valparaíso, 2020, No 16, 137-167

(c) $(1)(9)$ CC BY-NC-ND 
Numerus surdus y armonía musical. Sobre el temperamento igual y el fin del reinado pitagórico de los números Lianggi Espinoza; Juan Redmond; Pablo César Palacios Torres; Ismael Cortez Aguilera

García Pérez, Amaya (2014). El temperamento igual en los instrumentos de cuerda con trastes. En Amaya Sara García Pérez, Paloma Otaola González (coord.), Salinas, Francisco de. Música, teoría y matemática en el Renacimiento, pp. 61-89. Ediciones Universidad de Salamanca.

García Pérez, Amaya (2013). Francisco de Salinas y la teoría musical renacentista. En Amaya García Pérez y Bernardo García-Bernalt Alonso (eds.), De Musica libri septem de Francisco de Salinas, pp. 42-92. Salamanca: Ediciones Universidad de Salamanca.

Garrido, Fuensanta (2012). Lo que vibra es el yunque: Análisis de Nicom. Harm. VI, pp. 245.18248.26. Cuadernos de filología clásica. Estudios griegos e indoeuropeos, 22, 127-140. http://dx.doi.org/10.5209/rev_CFCG.2012.v22.39067

Garrido, Fuensanta (2016). Los teóricos menores de la música griega: Euclides el Geómetra, Nicómaco de Gerasa y Gaudencio el Filósofo. Barcelona: Cérix.

Gómez, Maricarmen (1992). Some Precursors of the Spanish Lute School. Early Music, 20(4), 583-593. Retrieved December 16, 2020, from http://www.jstor.org/stable/3128024

González, Alfonso H. (2014). La evitación de los números irracionales en la teoría musical antigua y sus consecuencias. La Gaceta de la RSME, 17(4), pp. 743-764.

Huffman, Carl (2018). Pythagoras. The Stanford Encyclopedia of Philosophy, Edward N. Zalta (ed.). URL: https://plato.stanford.edu/archives/win2018/entries/pythagoras/

Iamblichus (1989). On the Pythagorean Way of Life. Liverpool: Liverpool University Press.

Lindley, Mark y Turner-Smith, Ronald (1993). Mathematical Models of Musical Scales. Bonn: Verlag für systematische Musikwissenschaft $\mathrm{GmbH}$.

Mersenne, Marin (1637). Harmonie universelle, Tomo 2, Paris: Pierre Ballard.

Paniagua, Carlos (2018). El laúd árabe medieval: historia y construcción. Madrid: Ediciones Polifemo.

Pope, Isabel (1961). La vihuela y su música en el ambiente humanístico. Nueva Revista De Filología Hispánica, 15(3-4), 364-376.

Rasch, Rudolf (2008). Simon Stevin and the Calculation of Equal Temperament. En Philippe Vendrix (ed.), Music and Mathematics, pp. 253-320. Turnhout: Brepols Publishers. https:// doi.org/10.1484/M.EM-EB.3.3286

Rossi, Paolo (2009). I Filosofi e le Macchine. Milano: Feltrinelli.

Salinas, Francisco (1577). De música libri septem. Salamanca: Mathias Gastius.

Stevin, Simon (1585). L'Arithmétique. Leiden: L'Imprimerie de Christophle Plantin.

Strunk, Oliver (1950). Source Readings in Music History. From Classical Antiquity through the Romantic Era. New York: Norton \& Company Inc.

Tannery, Paul (1915). Du rôle de la musique grecque dans le développement de la musique pure. En Paul Tannery, Mémoires Scientifiques, Vol. 3, pp.68-87. Toulouse: Privat and Paris, Gauthier-Villars. 
Numerus surdus y armonía musical. Sobre el temperamento igual y el fin del reinado pitagórico de los números Lianggi Espinoza; Juan Redmond; Pablo César Palacios Torres; Ismael Cortez Aguilera

Tinctoris, Johannes (1917). De inventione et usu musicae. Regensburg: Friedrich Pustet.

Van Wymeersch, Brigitte (2008). Qu'entend-on par «nombre sourd»? En Philippe Vendrix (ed.), Music and Mathematics, pp. 97-110. Turnhout: Brepols Publishers.

Zarlino, Gioseffo (1558). Istitutioni harmoniche. Venecia: Francesco dei Francheschi Senese.

Zarlino, Gioseffo (1571). Dimonstrationi harmoniche. Venecia: Francesco Senese.

Zarlino, Gioseffo (1588). Sopplimenti musicali. Venecia: Francesco dei Francheschi Senese. 
